\title{
Qualidade de sementes de soja após a inoculação de biológicos em campo
}

\author{
Quality of soybean seeds after inoculation of biologicals in the field
}

Calidad de las semillas de soja después de la inoculación de biológicos en el campo

Roney Eloy Lima

ORCID: https://orcid.org/0000-0002-7588-7895 Universidade Federal de Mato Grosso do Sul, Brasil E-mail: roney.eloylima@yahoo.com.br Jorge González Aguilera

ORCID: https://orcid.org/0000-0002-7308-0967 Universidade Federal de Mato Grosso do Sul, Brasil E-mail: j51173@yahoo.com

Alan Mario Zuffo

ORCID: https://orcid.org/0000-0001-9704-5325 Universidade Federal de Mato Grosso do Sul, Brasil E-mail: alan_zuffo@hotmail.com

Charline Zaratin Alves

ORCID: https://orcid.org/0000-0001-6228-078X Universidade Federal de Mato Grosso do Sul, Brasil E-mail: charline.alves@ufms.br

Rafael Felippe Ratke

ORCID: https://orcid.org/0000-0001-6930-3913 Universidade Federal de Mato Grosso do Sul, Brasil E-mail: rafael.ratke@ufms.br

Graziely Alves Nogueira

ORCID: https://orcid.org/0000-0001-6985-3317 Universidade Federal de Mato Grosso do Sul, Brasil E-mail: graziely.nogueira@hotmail.com

Anielle Verzotto Teixeira

ORCID: https://orcid.org/0000-0003-3642-0923 Universidade Federal de Mato Grosso do Sul, Brasil

E-mail: anielliverzottoteixeira@ hotmail.com

Ana Carina da Silva Cândido

ORCID: https://orcid.org/0000-0002-9230-4807 Universidade Federal de Mato Grosso do Sul, Brasil E-mail: ana.candido@ufms.br

\begin{abstract}
Resumo
A combinação de bactérias fixadoras de nitrogênio e fungos biocontroladores que interferem no desenvolvimento de plantas pode constituir uma ferramenta que permita promover uma melhor qualidade de sementes obtidas sob estas combinações. O objetivo deste trabalho foi verificar a interação de bioprodutos a base de Bradyrhizobium japonicum, Azospirillum brasilense, Trichoderma asperrellum e BioAx aplicados no solo visando melhorar a qualidade de sementes em cultivares de soja. O delineamento experimental utilizado foi inteiramente casualizado em esquema fatorial $3 \times 4$, com três repetições. Os tratamentos consistiram em três cultivares de soja [Brasmax Foco IPRO, Brasmax Desafio IPRO e Brasmax Bônus IPRO] e quatro biológicos [T1- Azospirillum brasilense + Bradyrhizobium japonicum, T2- BioAx, T3- Trichoderma asperrellum, e T4- a combinação dos três biológicos]. Avaliou-se: a primeira contagem de germinação, germinação, condutividade elétrica, massa seca da parte área e de raízes de plântulas e teste de tetrazólio (vigor e viabilidade). Os resultados mostraram que as respostas obtidas foram dependentes das cultivares avaliadas, e que Brasmax Foco IPRO e Brasmax Desafio IPRO apresentaram maior qualidade fisiológica das sementes. $\mathrm{O}$ efeito dos biológicos dependem da cultivar da soja; em cultivares com maior qualidade fisiológica o efeito do biológico não é evidente. Entretanto, em cultivares com menor qualidade fisiológica (Brasmax Bônus IPRO), o efeito dos biológicos é mais evidente, constituindo assim uma excelente alternativa de se melhorar a qualidade das sementes de soja com o uso combinado de biológicos.
\end{abstract}

Palavras-chave: Azospirillum brasilense; Bradyrhizobium japonicum; BioAx; Glycine max; Teste de tetrazólio; Trichoderma asperrellum.

\footnotetext{
Abstract

The combination of nitrogen-fixing bacteria and biocontrolling fungi that interfere with the development of plants can be a tool to promote a better quality of seeds obtained under these combinations. The objective of this work was to verify the interaction of bioproducts based on Bradyrhizobium japonicum, Azospirillum brasilense, Trichoderma
} 
asperrellum and BioAx applied to the soil to improve seed quality in soybean cultivars. The experimental design used was completely randomized in a $3 \times 4$ factorial scheme, with three replications. The treatments consisted of three soybean cultivars [Brasmax Foco IPRO, Brasmax Desafio IPRO and Brasmax Bonus IPRO] and four biological [T1Azospirillum brasilense + Bradyrhizobium japonicum, T2- BioAx, T3- Trichoderma asperrellum, and T4- the combination of the three biologicals]. It was evaluated: the first germination count, germination, electrical conductivity, dry mass of the area and seedling roots and tetrazolium test (vigor and viability). The results showed that the responses obtained were dependent on the cultivars evaluated, and that Brasmax Foco IPRO and Brasmax Desafio IPRO showed higher physiological seed quality. The effect of biologicals depends on the soybean cultivar; in cultivars with higher physiological quality, the biological effect is not evident. However, in cultivars with a lower physiological quality (Brasmax Bonus IPRO), the effect of biologicals is more evident, thus constituting an excellent alternative to improve the quality of soybean seeds with the combined use of biologicals.

Keywords: Azospirillum brasilense; Bradyrhizobium japonicum; BioAx; Glycine max; Tetrazolium test; Trichoderma asperrellum.

\section{Resumen}

La combinación de bacterias fijadoras de nitrógeno y hongos biocontroladores que interfieren con el desarrollo de las plantas puede ser una herramienta para promover una mejor calidad de semillas obtenidas bajo estas combinaciones. El objetivo de este trabajo fue verificar la interacción de bioproductos basados en Bradyrhizobium japonicum, Azospirillum brasilense, Trichoderma asperrellum y BioAx aplicado al suelo para mejorar la calidad de la semilla en cultivares de soja. El diseño experimental utilizado fue completamente al azar en un esquema factorial $3 \times 4$, con tres repeticiones. Los tratamientos consistieron en tres cultivares de soja [Brasmax Foco IPRO, Brasmax Desafio IPRO y Brasmax Bonus IPRO] y cuatro biológicos [T1- Azospirillum brasilense + Bradyrhizobium japonicum, T2- BioAx, T3- Trichoderma asperrellum y T4- la combinación de los tres biológicos]. Se evaluó: primer conteo de germinación, germinación, conductividad eléctrica, masa seca del área y raíces de plántula y prueba de tetrazolio (vigor y viabilidad). Los resultados mostraron que las respuestas obtenidas fueron dependientes de los cultivares evaluados, y que Brasmax Foco IPRO y Brasmax Desafio IPRO presentaron mayor calidad fisiológica de las semillas. El efecto de los biológicos depende del cultivo de soja; en cultivares de mayor calidad físiológica, el efecto biológico no es evidente. Sin embargo, en cultivares de menor calidad fisiológica (Brasmax Bonus IPRO), el efecto de los biológicos es más evidente, constituyendo así una excelente alternativa para mejorar la calidad de las semillas de soja con el uso combinado de biológicos.

Palabras clave: Azospirillum brasilense; Bradyrhizobium japonicum; BioAx; Glycine max; Prueba de tetrazolio; Trichoderma asperrellum.

\section{Introdução}

O Brasil é o maior produtor mundial de soja (Glicine max L. Merrill) e sua comercialização exerce grande importância econômica tanto para o abastecimento nacional quanto para exportação (CONAB, 2020). A cultura na safra 2019/2020, apresentou 36,9 milhões hectares de área cultivada e uma produção de 124,8 milhões de toneladas de grãos (CONAB , 2020). Para garantir a produtividade dos grãos de soja é essencial suprir as necessidades nutricionais do cultivo e, também o uso de sementes com qualidade fisiológica satisfatória. O nitrogênio (N) é um dos elementos mais exigidos pela soja, devido ao elevado teor de proteína nas sementes. A inoculação das sementes de soja com Bradyrhizobium japonicum possibilita fornecer $\mathrm{N}$ para a cultura sem necessidade de adubação mineral, proporcionando maior rendimento na produção de sementes com menor custo para o produtor (Basal \& Szabó, 2019).

As leguminosas obtêm $\mathrm{N}$ de duas formas, o nitrogênio mineral disponível no solo e pela fixação biológica do nitrogênio (FBN). A FBN supre as necessidades nutricionais de $\mathrm{N}$ em leguminosas em até 94\% do $\mathrm{N}$ requerido pela cultura (Hungria et al. 2007), reduzindo o custo com adubação, porém, aumenta o consumo de fotoassimilados causando a redução na produção e na concentração de óleo das sementes (Tamagno et al., 2018).

Um dos fatores necessários para alcançar altas produtividades e o sucesso no processo de FBN é a utilização de inoculante à base de bactérias B. japonicum em sementes de soja. Este inoculante promove aumento do número e peso dos nódulos e no número de vagens, resultando em maior produtividade dos grãos durante o cultivo (Jarecki et al., 2019). Esses resultados indicam aos inoculantes biológicos como excelentes ferramentas a serem empregadas para alcançar uma melhor produção, garantindo a boa nutrição da planta sendo aplicados no tratamento de sementes (TS) ou em inoculações no solo. 
Azospirillum sp. apresenta grande importância agronômica por promover o crescimento, desenvolvimento e produtividade de centenas de espécies de vegetais atuando na FBN (Cassán \& Diaz-Zorita, 2016, Galindo et al., 2018). Ele promove a produção de fitohôrmonios (auxinas, giberelinas, citokininas, etileno e ABA) e aumento do crescimento radicular amplificando a capacidade de absorção de água e nutrientes pelas plantas (Cassán \& Diaz-Zorita, 2016). A. brasilense tem sido empregado na co-inoculação com o B. japonicum aumentando a eficiência da FBN, obtenção de água e nutrientes, possibilitando maior rendimento na produção de sementes de soja devido ao maior incremento no número de vagens e no peso de 1000 grãos (Chibeba et al., 2015, Ferri et al., 2017, Galindo et al., 2018, Rego et al., 2018), assim como, tem sido observada a melhora na qualidade da semente (Zuffo et al., 2016). Apesar da inoculação de A. brasiliense promover diversos processos biológicos que contribuem para o crescimento das plantas de soja, sua inoculação isolada não é favorável para a produção de sementes (Brum et al., 2018).

A utilização de fungos do gênero Trichoderma na cultura da soja contribui no biocontrole de fungos fitopatogênicos (Mesquita et al., 2017), além de atuarem como promotores de crescimento de plantas (Carvalho Filho et al., 2018) e favorecer o desenvolvimento aumentando a produtividade de sementes (Marra et al., 2019). Isolados de T. harzianum apresentaram capacidade de biocontrole contra Macrophomina phaseolina responsável pela podridão radicular do carvão da soja (Khaledi \& Taheri, 2016). No controle de Sclerotinia sclerotiorum, as cepas de Trichoderma (T. koningiopsis, T. asperelloides, T. atroviride e $T$. virens) foram eficientes garantindo a sanidade das plantas de soja além de melhorar o crescimento da parte aérea (Haddad et al., 2014).

O BioAx é outro produto biológico, composto por uma mistura de bactérias que é recomendado para promover na planta uma melhor assimilação de nutrientes segundo os obtentores desse produto (AMK, 2019), entretanto, poucos trabalhos têm sido publicados mostrando esses benefícios. Aguilera et al. (2020c) relatam seu uso e a promoção do crescimento de cultivares de soja ao considerar o número de grãos por vagens e por planta, e o estimulo em favorecer a produção de grão de peneira $\emptyset 6.0$ e $\varnothing 5.0$.

Alternativamente, a combinação de bactérias FBN e fungos biocontroladores que interferem no desenvolvimento de espécies de plantas pode constituir uma ferramenta na recuperação do equilíbrio da biota do solo além de promover um maior rendimento das culturas submetidas a estas combinações. O manejo da soja no campo determina a qualidade fisiológica das sementes produzidas. A aplicação de diversos produtos biológicos na cultura da soja é realizada com diferentes fins, porém, os efeitos deles sob a qualidade das sementes não tem sido avaliada. Assim, objetivou avaliar se a interação de bioprodutos a base de Bradyrhizobium japonicum, Azospirillum brasilense, Trichoderma asperrellum e BioAx, aplicados no solo, melhoram a qualidade de sementes de soja.

\section{Material e Métodos}

\subsection{Condições experimentais}

A presente pesquisa é de campo e laboratorial, seguindo os fundamentos para este tipo de pesquisa segundo recomendações de Pereira et al. (2018). O experimento foi conduzido em área experimental da Universidade Federal de Mato Grosso do Sul (UFMS), Campus de Chapadão do Sul, Mato Grosso do Sul (MS) (1846'17,9" de latitude sul, 52³7'25,0" de longitude oeste e altitude média de 810 m) na safra 2018/2019. O clima da região está de acordo com a classificação Köppen, do tipo chuvoso tropical (Aw), com verão chuvoso e inverno seco, com precipitação, temperatura média e umidade relativa anual de $1.261 \mathrm{~mm}, 23,97^{\circ} \mathrm{C}$ e $64,23 \%$, respectivamente, o acumulado de precipitação durante a condução do experimento foi de $1.150 \mathrm{~mm}$. 
O solo da área experimental é um Latossolo vermelho distrófico com textura argilosa. Antes de iniciar o experimento, amostras de solo foram coletadas a uma profundidade de $0.00-0.20 \mathrm{~m}$ e as principais propriedades químicas foram determinadas (Tabela 1).

Tabela 1. Principais propriedades químicas do solo usado no experimento.

\begin{tabular}{|c|c|c|c|c|c|c|c|c|c|}
\hline $\mathrm{pH}$ & MO & PMehlich $^{-1}$ & $\mathrm{H}+\mathrm{Al}$ & $\mathrm{Al}^{3+}$ & $\mathrm{Ca}^{2+}$ & $\mathrm{Mg}^{2+}$ & $\overline{\mathrm{K}^{+}}$ & CTC & $\mathrm{V}$ \\
\hline $\mathrm{CaCl}_{2}$ & $\mathrm{~g} \mathrm{dm}^{-3}$ & $\mathrm{mg} \mathrm{dm}^{-3}$ & $-\cdots$ & ------- & $\mathrm{lc} \mathrm{dm}$ & - & ----- & & $\%$ \\
\hline 4.3 & 22.8 & 12.8 & 5.7 & 0.3 & 2.20 & 0.40 & 0.27 & 8.6 & 33.5 \\
\hline
\end{tabular}

MO: Matéria orgânica. CTC: Capacidade de troca catiônica em pH 7.0. V: Saturação da base. Fonte: Dados da pesquisa

A correção da acidez do solo para elevar a saturação por base para $60 \%$ foi realizada com a aplicação superficial de 2,5 $\mathrm{t} \mathrm{ha}^{-1}$ de calcário (CaO: 29\%, MgO: 20\%, PRNT: 90,1\%, PN: 101,5\%). O calcário foi aplicado 60 dias antes da implantação do experimento. A semeadura da soja foi realizada mecanicamente, com auxílio de uma semeadora-adubadora, com distribuição de 13 sementes por metro, com espaçamento entre linhas de $0,45 \mathrm{~m}$. A adubação de base foi constituída por $150 \mathrm{~kg} \mathrm{ha}^{-1}$ de $\mathrm{P}_{2} \mathrm{O}_{5}$, cuja fonte era superfosfato simples. Aos 40 dias após a emergência (DAE), foi realizada a aplicação de adubação foliar dos produtos Actilasa ZM (Zn a $50.22 \mathrm{~g} \mathrm{~L}^{-1}$; S a $41.65 \mathrm{~g} \mathrm{~L}^{-1}$; Mn a $30.01 \mathrm{~g} \mathrm{~L}^{-1}$ ) e Racine (Mo a $108.75 \mathrm{~g} \mathrm{~L}^{-1}$; Co a $10.88 \mathrm{~g} \mathrm{~L}^{-1}$; Carbono total $123.25 \mathrm{~g} \mathrm{~L}^{-1}$ ), as doses foram de $1 \mathrm{~L} \mathrm{ha}^{-1} \mathrm{e} 120 \mathrm{~mL}$ para ha- ${ }^{-1}$, respectivamente.

\subsection{Delineamento experimental}

O delineamento experimental utilizado no campo foi o fatorial de $3 \times 4$, com três repetições. O fator cultivar foi constituído por três cultivares de soja [BRASMAX Foco IPRO - hábito de crescimento indeterminado, ciclo médio de 109 dias, grupo de maturação 7.2, BRASMAX Desafio IPRO - hábito de crescimento indeterminado, ciclo médio de 113 dias, grupo de maturação 7.4 e BRASMAX Bônus IPRO - hábito de crescimento indeterminado, ciclo médio de 121 dias, grupo de maturação 7.9] e o fator biológico foi constituído por quatro biológicos: T1- Azospirillum brasilense (Cepas AbV5 e AbV6) + Bradyrhizobium japonicum (SEMIA 5079 e 5080) ambos na doses de $2 \mathrm{~L} \mathrm{ha}^{-1}$ empregados como controle, T2- BioAx (conjunto de bactérias não especificado pelo fabricante) na doses de $5 \mathrm{~L} \mathrm{ha}^{-1}$, T3- Trichoderma asperrellum (Isolado SF 04) na doses de $100 \mathrm{~g} \mathrm{ha}^{-1}$, e T4- a combinação dos três biológicos empregando as mesmas doses deles isoladamente. Os biológicos foram aplicados empregando bomba costal de $20 \mathrm{~L}$ nas doses recomendadas por tratamento e fazendo a diluição dos produtos comerciais em água.

As sementes foram semeadas sem nenhum tratamento, a fim de garantir o efeito isolado dos biológicos aplicados após a semeadura numa área com histórico de manejo em sistema de plantio direto (SPD). Cada unidade experimental foi constituída de 6 linhas de $3 \mathrm{~m}$ de comprimento, sendo as duas linhas laterais de cada lado desconsideradas e, 0,50 m em cada extremidade, constituindo assim a área útil utilizada em todas as análises de nosso experimento de 2 linhas de $2 \mathrm{~m}$ cada. Durante todo o ciclo da cultura, o controle de plantas daninhas, pragas e doenças nocivas foi realizado com produtos químicos, de acordo com a necessidade e a recomendação para a cultura.

\subsection{Variáveis mesuradas na qualidade fisiológica das sementes}

As sementes das três cultivares colhidas no final do experimento de campo foram empregadas nos testes de qualidade fisiológica em laboratório. Inicialmente determinou-se o teor de umidade das sementes a partir da pesagem de $10 \mathrm{~g}$ de amostra em duas repetições, colocadas em estufa a $105^{\circ} \mathrm{C} \pm 1{ }^{\circ} \mathrm{C}$ durante $24 \mathrm{~h}$; em seguida as amostras foram retiradas e colocadas em dessecador para posterior pesagem (Brasil, 2009). 
Foram avaliadas as seguintes variáveis: primeira contagem da germinação (PCG); germinação (GERM); comprimento da parte área (CPA); comprimento da raiz (CR); massa seca da parte área (MSPA); massa seca de raiz (MSR); condutividade elétrica (CE) e tetrazólio [vigor (VIG); viabilidade (VIA)].

Para o teste de germinação foram empregadas quatro repetições de 50 sementes, distribuídos em rolos de papel tipo germitest, umedecido com água destilada na proporção 2,5 vezes a massa do papel não hidratado, em germinador tipo B.O.D. regulado a $25^{\circ} \mathrm{C} \pm 2{ }^{\circ} \mathrm{C}$. As avaliações foram realizadas no $5^{\circ}$ (PCG) e $8^{\circ}$ dia (GERM) após a instalação do teste por meio da contagem de plântulas normais (Brasil, 2009).

Para avaliar o crescimento (CPA e CR) e a massa seca (MSPA e MSR) de plântulas de soja, foram utilizadas quatro repetições de 20 sementes e colocadas para germinar nas mesmas condições do teste de germinação. Aos sete dias, as plântulas foram retiradas do germinador e separada a parte área (hipocótilo e cotilédone) da raiz, aferida o comprimento de ambas as partes $(\mathrm{CPA}$ e $\mathrm{CR})$ de modo individual com auxílio de uma régua milimetrada $(\mathrm{cm})$. Após, as amostras foram colocadas em embalagem de papel kraft em estufa de ar forçado a $65^{\circ} \mathrm{C}$ durante três dias. Ao término desse período, as amostras formam pesadas (mg) em balança de precisão (0,001 g) (Brasil, 2009).

A CE foi aferida em quatro repetições por tratamento, cada uma contendo 25 sementes, pesadas em uma balança de precisão de $0,001 \mathrm{~g}$ e colocadas em copos plásticos com $75 \mathrm{~mL}$ de água destilada e após acondicionados em $\mathrm{BOD}$ a $25^{\circ} \mathrm{C}$, por 24h. Após esse período foi feita a leitura de CE da solução de embebição com condutivímetro digital da marca Conductivity Meter modelo CD-4301. Os resultados foram expressos em $\mu \mathrm{S} \mathrm{cm}^{-1} \mathrm{~g}^{-1}$ conforme a metodologia descrita por Marcos Filho et al. (1987).

Para o teste de tetrazólio, duas repetições de 50 sementes foram submetidas ao pré-umedecimento em papel germitest durante 16 horas a $25^{\circ} \mathrm{C}$, e em seguida foram submetidos a solução de tetrazólio a 0,075\%, mantidos durante três horas a 35 ${ }^{\circ} \mathrm{C}$ na ausência de luz. Após este período, as sementes foram lavadas em água corrente e avaliadas as variáveis VIG e VIA de acordo com a metodologia estabelecida por França-Neto e Krzyzanowski (2018).

\subsection{Análises estatísticas}

Os dados experimentais foram submetidos aos testes de verificação dos pressupostos de normalidade e homogeneidade de variância. Posteriormente, foi efetuada à análise de variância (ANOVA) e, quando significativos, as médias foram comparadas pelo teste de Tukey a 5\% de probabilidade, utilizando o programa estatístico GENES (Cruz, 2013). Correlações de Pearson foram estabelecidas entre as variáveis e com elas estabelecida uma rede de correlações, e para melhor interpretar os dados uma análise de variáveis canônicas foi realizada empregando o programa RBio (Bhering, 2017). Gráficos foram gerados no SigmaPlot 11.0 para Windows (Systat Software, Inc., San José, CA, EUA).

\section{Resultados e Discussão}

A Tabela 2 apresenta os resultados obtidos ao avaliar o uso dos biológicos e sua influência na qualidade das sementes de soja. A interação entre os fatores avaliados (cultivares $\mathrm{x}$ biológicos) apresentaram resultados altamente significativos $(P<0.01)$ para a maioria das variáveis, com a exceção de MSPA e MSR que não manifestaram interações significativas. Os CV que são uma medida da precisão dos dados obtidos mostram valores adequados para experimentos de laboratórios com sementes de soja, como os obtidos por Rego et al. (2018) ao avaliar a qualidade de soja como resultado da co-inoculação de Bradyrhizobium spp. e Azospirillum brasilense. 
Tabela 2. Resumo da ANOVA obtido ao avaliar o efeito de biológicos aplicados no campo na qualidade de sementes de soja.

\begin{tabular}{|c|c|c|c|c|c|c|c|c|c|c|}
\hline \multirow[b]{2}{*}{ Fatores } & \multirow[b]{2}{*}{ GL } & \multicolumn{9}{|c|}{ Quadrados médios } \\
\hline & & $\begin{array}{c}\text { PCG1 } \\
(\%)\end{array}$ & $\begin{array}{c}\text { GERM } \\
(\%)\end{array}$ & $\begin{array}{l}\text { CPA } \\
(\mathrm{cm})\end{array}$ & $\begin{array}{l}\mathrm{CR} \\
(\mathrm{cm})\end{array}$ & $\begin{array}{c}\text { MSPA } \\
(\mathrm{g})\end{array}$ & $\begin{array}{c}\text { MSR } \\
(\mathrm{g})\end{array}$ & $\begin{array}{c}\mathrm{CE} \\
(\mu \mathrm{S} \mathrm{cm}-1 \mathrm{~g}- \\
1)\end{array}$ & $\begin{array}{l}\text { VIG } \\
(\%)\end{array}$ & $\begin{array}{l}\text { VIA } \\
(\%)\end{array}$ \\
\hline Cultivar (C) & 2 & $8459 * *$ & $5120 * *$ & $209 * *$ & $625 * *$ & $81 * *$ & $141 * *$ & $784 * *$ & $1842 * *$ & $198 * *$ \\
\hline Biológico (B) & 3 & $661 * *$ & $412 * *$ & 0,5 & $5 * *$ & 10 & 5 & $261 *$ & $285 * *$ & 19 \\
\hline $\mathrm{CxB}$ & 6 & $796 * *$ & $642 * *$ & $2 * *$ & $6^{* *}$ & 13 & 2 & $349 * *$ & $271 * *$ & $123 * *$ \\
\hline Residual & 165 & 32 & 20 & 0,4 & 0,7 & 8 & 2 & 99 & 33 & 12 \\
\hline $\mathrm{CV}(\%)$ & & 6,4 & 4,92 & 11,02 & 12,39 & 17,97 & 30,72 & 10,21 & 6,41 & 3,68 \\
\hline
\end{tabular}

${ }^{1}$ Primeira contagem da germinação (PCG); germinação (GERM); comprimento da parte área (CPA); comprimento da raiz (CR); massa seca da parte área (MSPA); massa seca de raiz (MSR); condutividade elétrica (CE) e tetrazólio [vigor (VIG); viabilidade (VIA)]. * $(\mathrm{P}<0.05)$ e ** $(\mathrm{P}<0.01)$ significativos pelo teste F. Fonte: Dados da pesquisa

A ausência de interação para as variáveis MSPA e MSR é apresentada na Tabela 3. Os resultados mostram que os biológicos não apresentaram diferenças significativas para estas variáveis. Este resultado evidencia que o emprego de qualquer um dos biológicos testados não promoveu um ganho significativo de matéria seca em nenhum dos órgãos avaliados (parte aérea e raiz). Porém, para estas mesmas variáveis (MSPA e MSR) as cultivares se diferenciaram de modo significativo $(P<0.05)$, com o mesmo comportamento para ambas as variáveis e superioridade das cultivares Brasmax Bônus IPRO e Brasmax Desafio IPRO sob a cultivar Brasmax Foco IPRO. Igual comportamento foi relatado por Aguilera et al. (2020a) ao avaliar as mesmas cultivares, o que evidencia que essas diferenças encontradas estão ligadas as características genéticas das cultivares manifestadas nas condições experimentais.

O desdobramento das interações significativas para cultivares x biológicos é apresentada na Tabela 4. Ao avaliar o efeito dos biológicos dentre as cultivares, observa-se que a cultivar Brasmax Foco IPRO não apresentou diferença em relação a utilização dos diferentes tratamentos biológicos para nenhuma das variáveis (Tabela 4).

Tabela 3. Médias das variáveis massa seca da parte área (MSPA) e da raiz (MSR) obtidas ao avaliar o efeito de biológicos aplicados no campo na qualidade de sementes de soja.

\begin{tabular}{lll}
\hline Cultivares & MSPA $(\mathrm{g})$ & MSR $(\mathrm{g})$ \\
\hline Brasmax Foco IPRO & $14,43 \mathrm{~b}$ & $3,55 \mathrm{~b}$ \\
Brasmax Desafio IPRO & $15,67 \mathrm{a}$ & $6,08 \mathrm{a}$ \\
Brasmax Bônus IPRO & $16,68 \mathrm{a}$ & $6,17 \mathrm{a}$ \\
\hline Biológicos & & \\
\hline T1 & & $4,80^{\mathrm{NS}}$ \\
T2 & $14,97^{\mathrm{NS}}$ & 5,36 \\
T3 & 15,61 & 5,45 \\
T4 & 15,73 & 5,45 \\
\hline
\end{tabular}

!(T1): Azospirillum brasilense + Bradyrhizobium japonicum, (T2): BioAx, (T3): Trichoderma asperrellum, (T4) soma de $1+2+3$. Médias seguidas da mesma letra na coluna não diferem estatisticamente pelo teste de Tukey ao $5 \%$. Fonte: Dados da pesquisa.

A cultivar Brasmax Desafio IPRO apresentou diferença em relação ao comprimento da raiz, onde os tratamentos com T2 e T3 apresentaram os maiores resultados e nos testes de tetrazólio para vigor e viabilidade, onde em ambas as variáveis o tratamento com T1 apresentou resultado inferior aos demais. A cultivar Brasmax Bônus IPRO por sua vez, apresentou diferenças significativas em relação ao emprego dos diferentes biológicos para todas as variáveis (Tabela 4), apresentando os melhores resultados para a qualidade das sementes de modo geral no tratamento T4 (combinação de todos os biológicos) e a pior resposta foi encontrada no tratamento T1 (controle) com A. brasilense + B. japonicum. A cultivar Brasmax Bônus IPRO em comparação com as outras duas cultivares foi a que apresentou os piores desempenhos para todas as variáveis que 
manifestaram interação significativa independente do biológico empregado (Tabela 4). Evidenciando assim, o pior desempenho quanto a qualidade integral das sementes se consideramos as sete características ligadas a qualidade de sementes de soja testadas, e com isso padrão inadequado para comercialização de sementes e plantio (APPS, 2013).

Tabela 4. Desdobramento da interação de cultivares x biológicos avaliadas em sementes de soja.

\begin{tabular}{|c|c|c|c|}
\hline \multirow[t]{2}{*}{ Biológicos } & Brasmax Foco IPRO & Brasmax Desafio IPRO & Brasmax Bônus IPRO \\
\hline & \multicolumn{3}{|c|}{ Primeira contagem da germinação $(\%)$} \\
\hline $\mathrm{T} 1^{\frac{1}{4}}$ & $95,75 \mathrm{Aa}$ & $95,13 \mathrm{Aa}$ & $66,00 \mathrm{Cb}$ \\
\hline $\mathrm{T} 2$ & $96,50 \mathrm{Aa}$ & $95,25 \mathrm{Aa}$ & $65,62 \mathrm{Cb}$ \\
\hline $\mathrm{T} 3$ & $96,50 \mathrm{Aa}$ & $95,88 \mathrm{Aa}$ & $81,13 \mathrm{Bb}$ \\
\hline \multirow[t]{2}{*}{$\mathrm{T} 4$} & $94,50 \mathrm{Aa}$ & $94,50 \mathrm{Aa}$ & $89,63 \mathrm{Ab}$ \\
\hline & \multicolumn{3}{|c|}{ Germinação (\%) } \\
\hline $\mathrm{T} 1$ & $97,63 \mathrm{Aa}$ & $97,38 \mathrm{Aa}$ & $70,13 \mathrm{Db}$ \\
\hline $\mathrm{T} 2$ & $97,63 \mathrm{Aa}$ & 96,75 Aa & $75,50 \mathrm{Cb}$ \\
\hline $\mathrm{T} 3$ & $97,25 \mathrm{Aa}$ & $96.75 \mathrm{Aa}$ & $87,63 \mathrm{Bb}$ \\
\hline \multirow[t]{2}{*}{$\mathrm{T} 4$} & 95,75 Aa & $95,25 \mathrm{Aa}$ & $92,00 \mathrm{Aa}$ \\
\hline & \multicolumn{3}{|c|}{ Comprimento da parte aérea $(\mathrm{cm})$} \\
\hline $\mathrm{T} 1$ & $6,08 \mathrm{Ab}$ & $6,83 \mathrm{Aa}$ & $3,08 \mathrm{Bc}$ \\
\hline $\mathrm{T} 2$ & $6,27 \mathrm{Ab}$ & $7,12 \mathrm{Aa}$ & $3,05 \mathrm{Bc}$ \\
\hline $\mathrm{T} 3$ & $6,19 \mathrm{Ab}$ & $6,82 \mathrm{Aa}$ & $3,65 \mathrm{Ac}$ \\
\hline \multirow[t]{2}{*}{$\mathrm{T} 4$} & $5,76 \mathrm{Ab}$ & $6,87 \mathrm{Aa}$ & $4,00 \mathrm{Ac}$ \\
\hline & \multicolumn{3}{|c|}{ Comprimento da raiz $(\mathrm{cm})$} \\
\hline $\mathrm{T} 1$ & $5,90 \mathrm{Ab}$ & $9,65 \mathrm{Ba}$ & $3,43 \mathrm{Cc}$ \\
\hline $\mathrm{T} 2$ & $6,04 \mathrm{Ab}$ & $10,99 \mathrm{Aa}$ & $3,72 \mathrm{BCc}$ \\
\hline $\mathrm{T} 3$ & $5,81 \mathrm{Ab}$ & $10,26 \mathrm{ABa}$ & $4,45 \mathrm{Bc}$ \\
\hline \multirow[t]{2}{*}{$\mathrm{T} 4$} & $5,70 \mathrm{Ab}$ & $10,22 \mathrm{Ba}$ & $5,37 \mathrm{Ab}$ \\
\hline & \multicolumn{3}{|c|}{ Condutividade elétrica $\left(\mu \mathrm{S} \mathrm{cm}^{-1} \mathrm{~g}^{-1}\right)$} \\
\hline T1 & $95,18 \mathrm{Ab}$ & $96,52 \mathrm{Aab}$ & $104,13 \mathrm{Aa}$ \\
\hline $\mathrm{T} 2$ & $91,28 \mathrm{Ab}$ & $101,95 \mathrm{Aa}$ & $105,23 \mathrm{Aa}$ \\
\hline $\mathrm{T} 3$ & $93,33 \mathrm{Aa}$ & $99,36 \mathrm{Aa}$ & $93,06 \mathrm{Ba}$ \\
\hline \multirow[t]{2}{*}{$\mathrm{T} 4$} & $92,47 \mathrm{Aab}$ & $100,65 \mathrm{Aa}$ & $91,50 \mathrm{Bb}$ \\
\hline & \multicolumn{3}{|c|}{ Tetrazólio - Vigor (\%) } \\
\hline $\mathrm{T} 1$ & $93,25 \mathrm{Aa}$ & $83,00 \mathrm{Bb}$ & $81,75 \mathrm{Bb}$ \\
\hline $\mathrm{T} 2$ & $96,25 \mathrm{Aa}$ & $93,25 \mathrm{Aa}$ & $77,00 \mathrm{Bb}$ \\
\hline $\mathrm{T} 3$ & 94,75 Aa & $91,25 \mathrm{Aab}$ & $88,5 \mathrm{Ab}$ \\
\hline \multirow[t]{2}{*}{$\mathrm{T} 4$} & $93,00 \mathrm{Aa}$ & $91,75 \mathrm{Aab}$ & $87,25 \mathrm{Ab}$ \\
\hline & \multicolumn{3}{|c|}{ Tetrazólio - Viabilidade (\%) } \\
\hline $\mathrm{T} 1$ & $96,00 \mathrm{Aa}$ & $91,25 \mathrm{Bb}$ & $95,00 \mathrm{Aa}$ \\
\hline $\mathrm{T} 2$ & $98,25 \mathrm{Aa}$ & $96,50 \mathrm{Aa}$ & $88,75 \mathrm{Bb}$ \\
\hline $\mathrm{T} 3$ & $96,25 \mathrm{Aa}$ & $95,25 \mathrm{Aa}$ & $93,75 \mathrm{Aa}$ \\
\hline $\mathrm{T} 4$ & $95,25 \mathrm{Aa}$ & $97,00 \mathrm{Aa}$ & $94,25 \mathrm{Aa}$ \\
\hline
\end{tabular}

!(T1): Azospirillum brasilense + Bradyrhizobium japonicum, (T2): BioAx, (T3): Trichoderma asperrellum, (T4) soma de $1+2+3$. Médias seguidas da mesma letra maiúscula na coluna e minúsculas na linha, não diferem estatisticamente pelo teste de Tukey ao 5\%. Fonte: Dados da pesquisa

Na avaliação de primeira contagem de germinação e a germinação comportamento similar foi obtido, ao considerar a comparação das três cultivares, dentre de cada um dos biológicos aplicados, tendo destaque para as cultivares Brasmax Foco IPRO e Brasmax Desafio IPRO superiores a cultivar Brasmax Bônus IPRO independente do biológico aplicado. A cultivar Brasmax Bônus IPRO apresentou o menor percentual germinativo inferior a 80\%, padrão inadequado segundo a instituição normativa ${ }^{\circ} 45$ (APPS, 2013). Ao comercializar sementes de soja no Brasil é necessário percentual mínimo de 80\% de germinação. Para alcançar altos níveis de produtividade é necessário estabelecer uma população de plantas apropriada e uniforme isso só é possível utilizando sementes com altas taxas de germinação (Krzyzanowski, 2004). Sementes com 
percentual germinativo a baixo de $80 \%$ comprometem o desenvolvimento das plantas prejudicando seu estabelecimento competitivo no campo (França Neto et al., 2010).

Avaliação de comprimento da parte aérea e comprimento da raiz manifestaram igual comportamento independente do biológico empregado, revelando-se as maiores médias para a cultivar Brasmax Desafio IPRO e as menores médias para a cultivar Brasmax Bônus IPRO, demonstrando que houve um padrão proporcional para o crescimento do sistema radicular em relação a parte aérea das plantas, bem diferenciado $(P<0.05)$ entre as cultivares empregadas. O melhor desenvolvimento da parte área e de raízes em condições controladas pode ser um indicador de um melhor estabelecimento da cultura no campo, em se tratando de sementes com qualidade.

Testes de laboratório nem sempre correspondem com as respostas obtidas em campo, mas permitem ter melhor noção da qualidade das sementes e por isso a legislação brasileira os coloca como informações obrigatórias a serem fornecidas pelos obtentores de sementes (Brasil, 2009, APPS, 2013). Na mesma safra 2018/2019 na qual foram obtidas as sementes empregadas nos nossos experimentos, outros trabalhos (Aguilera et al., 2020a, da Silva et al., 2020) foram desenvolvidos ao avaliar tecnologias diferentes e empregar as mesmas cultivares Brasmax Desafio IPRO e Brasmax Bônus IPRO, evidenciando que a resposta depende das condições oferecidas no campo. Da Silva et al. (2020) ao avaliar o manejo de irrigação na cultura da soja em sistema de semeadura direta, sobre restos culturais de Brachiaria ruziziensis obteve melhor desempenho do Brasmax Desafio IPRO em relação a Brasmax Bônus IPRO com 3221 e $2642 \mathrm{~kg} \mathrm{ha}^{-1}$, respectivamente. Entretanto, Aguilera et al. (2020b) ao avaliar a influência das doses de pó de basalto obteve rendimentos de 4796 e $4940 \mathrm{~kg} \mathrm{ha}^{-1}$, respectivamente. Ambos os trabalhos foram feitos da mesma região e época de plantio, evidenciam-se assim as respostas que podemos ter nos mesmos genótipos ao se avaliar em experimentos diferentes, mostrando a divergência na resposta deles.

Zambiazzi et al. (2017) verificaram que os genótipos de soja apresentam variação na qualidade das sementes produzidas quando submetidas a diferentes ambientes de cultivo. A obtenção de sementes com elevada qualidade fisiológica e produtividade, depende da capacidade adaptativa do genótipo trabalhado e o ambiente de produção (França Neto et al., 2013).

$\mathrm{Na}$ avaliação da condutividade elétrica os tratamentos T1 e T2 apresentaram as menores médias na cultivar Brasmax Foco IPRO (95,18 e 91,28 $\mu \mathrm{S} \mathrm{cm}^{-1} \mathrm{~g}^{-1}$ respectivamente) e com isso a cultivar que manifesta a melhor resposta para esta variável. No T3 não apresentou diferença significativa entre as cultivares para CE e o T4 proporcionou a maior média na cultivar Brasmax Desafio IPRO com 100,65 $\mu \mathrm{S} \mathrm{cm}^{-1} \mathrm{~g}^{-1}$. Altos valores de CE estão associados a deterioro das membranas celulares. Consequentemente, menores valores acompanham uma melhor qualidade fisiológica das sementes (Brasil, 2009, Coradi et al., 2015).

O teste de tetrazólio possibilita determinar o vigor e viabilidade das sementes, bem como diagnosticar os problemas que causam a perda da qualidade, assim como, os danos provocados por umidade, danos mecânicos, danos provocados pelo ataque de insetos (percevejos) e danos causados por secagem (Brasil, 2009). A avaliação de vigor pelo tetrazólio nas sementes obtidas mostrou que em T1 a maior média esteve na cultivar Brasmax Foco IPRO (93.25\%), em T2 a menor média foi da cultivar Brasmax Bônus IPRO (77\%), em T3 e T4 as maiores médias foram da cultivar Brasmax Foco IPRO (94,75 e 93,00\%, respectivamente).

A avaliação de viabilidade pelo tetrazólio procura obter altos valores como indicadores de elevada qualidade das sementes (Brasil, 2009). Em T1 a menor média foi da cultivar Brasmax Desafio IPRO (91,25\%), em T2 a menor média foi da cultivar Brasmax Bônus IPRO (88.75\%), em T3 e T4 não houve diferença significativa entre as cultivares avaliadas. Sementes com um alto vigor e viabilidade manifestam uma maior capacidade adaptativa ao manifestar uma rápida germinação no campo (Soltani et al., 2006), promovendo vantagem competitiva sob as plantas daninhas (Dias et al., 2011). Tendo esse critério, podemos afirmar que a cultivar Brasmax Bônus IPRO foi a que manifestou o pior desempenho quando observado o vigor e as viabilidades das sementes independente do biológico aplicado. 
Com todas as características avaliadas e suas respectivas correlações um diagrama de redes foi construído (Figura 1). O diagrama de rede mostra a interação entre as características avaliadas destacando que as variáveis de germinação (GERM e PCG), qualidade (CE, VIG e VIA) e desenvolvimento (CPA, CR, MSPA e MSR) apresentaram forte interação entre elas. As correlações indicam que a qualidade das sementes de soja está diretamente ligada ao desempenho da germinação aliado a um bom desenvolvimento da planta. De todas as nove variáveis aferidas, a variável CE foi a única que apresentou correlações negativas com todas as variáveis. Esse efeito é observado pelos resultados registrarem a perda da qualidade das sementes avaliadas por isso seu resultado é inversamente proporcional às demais variáveis.

Figura 1. Diagrama de rede ilustrando as correlações de Pearson mais significativas encontradas entre variáveis representativas da germinação, desenvolvimento e qualidade de sementes de soja produzidas na Safra 2018/2019 em Chapadão do Sul, MS. Primeira contagem da germinação (PCG); germinação (GERM); comprimento da parte área (CPA); comprimento da raiz (CR); massa seca da parte área (MSPA); massa seca de raiz (MSR); condutividade elétrica (CE) e tetrazólio [vigor (VIG) e viabilidade (VIA)]. As linhas mais grossas vermelhas e verdes representam as correlações negativas e positivas mais altas (>0.6), respectivamente.

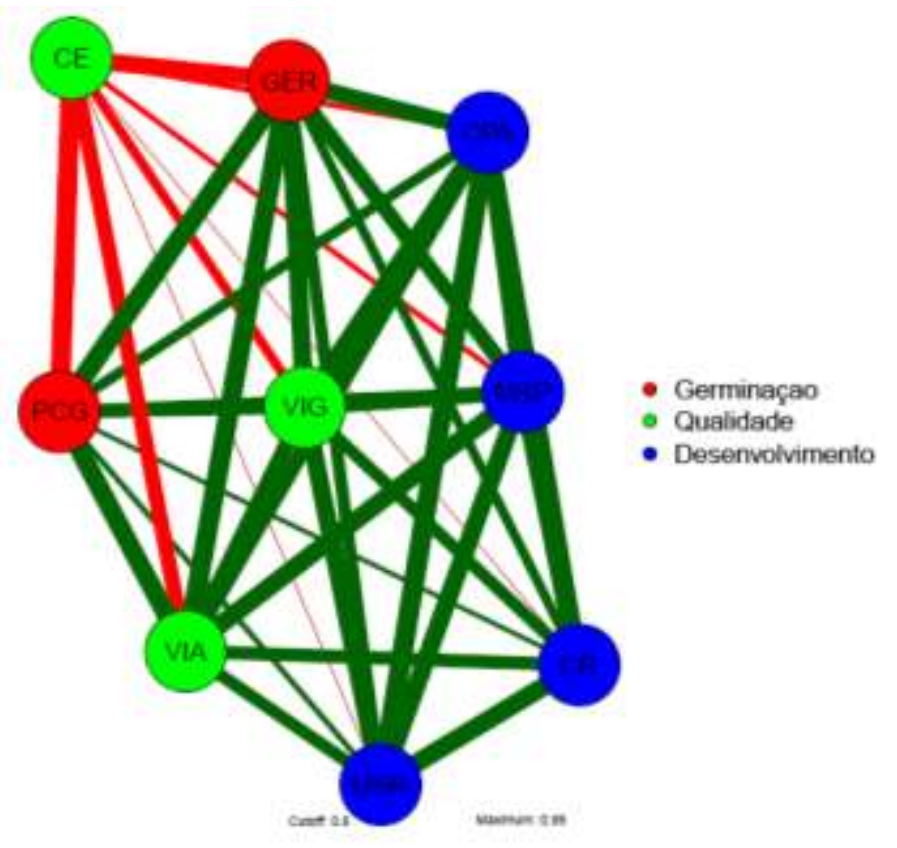

Fonte: Dados da pesquisa.

As duas primeiras variáveis canônicas explicam $100 \%$ (70,7\% relativa à primeira, e 29,3\% relativa à segunda, Figura 2A) e 96,4 \% (74,3\% relativa à primeira, e 22,1\% relativa à segunda, Figura 2B) ao considerar os dois fatores avaliados, cultivares e uso de biológicos, respectivamente. Assim, uma descrição adequada das respostas das cultivares e os biológicos pode ser feita utilizando-se apenas essas duas variáveis canônicas (Cruz et al., 2004). Ao considerar as cultivares usadas e sua relação com as variáveis mesuradas observa-se na Figura 2A que existe um contraste entre as respostas das cultivares, que complementa o entendimento da Tabela 4. Das cultivares a Brasmax Bônus IPRO apenas se associo com a MSPA, sendo assim a cultivar que pior desempenho em relação a qualidade de semente manifestou (Figura 2A). A cultivar Brasmax Foco IPRO foi a que se associou as características VIG, VIA, PCG, GERM e CPA e com isso essas variáveis foram as que mais contribuíram para que esta cultivar manifesta-se as melhores qualidades das sementes produzidas. A Brasmax Desafio IPRO, por sua vez, manifestou maior associação com CE, CR e MSR as quais determinaram a resposta obtida para esta cultivar (Figura 2A). Este tipo de abordagem tem sido muito útil na diferenciação e obtenção de cultivares de soja contrastantes para diferentes 
espaçamentos (Ferreira et al., 2020), seleção de genótipos de soja cultivados em várzea irrigada (dos Santos et al., 2011), e no tratamento químico de sementes e seu impacto sobre complexos de fitossanitários e elementos morfoagronômicos (dos Santos et al., 2019) entre outras aplicações.

Figura 2. Representação das duas primeiras variáveis canônicas, estabelecidos pela combinação linear de nove características representativas da germinação, desenvolvimento e qualidade de sementes de soja produzidas na Safra 2018/2019 em Chapadão do Sul, MS em relação aos fatores avaliados [cultivares (A) e biológicos (B)]. Primeira contagem da germinação (PCG); germinação (GERM); comprimento da parte área (CPA); comprimento da raiz (CR); massa seca da parte área (MSPA); massa seca de raiz (MSR); condutividade elétrica (CE) e tetrazólio [vigor (VIG) e viabilidade (VIA)]. Em A) os nomes Foco, Desafio e Bonus representam as cultivares: Brasmax Foco IPRO, Brasmax Desafio IPRO e Brasmax Bônus IPRO, respectivamente. Em B) os números 1, 2, 3 e 4 representam os tratamentos (T1): A. brasilense + B. japonicum, (T2): BioAx, (T3): $T$. asperrellum e (T4) soma de $1+2+3$, respectivamente.
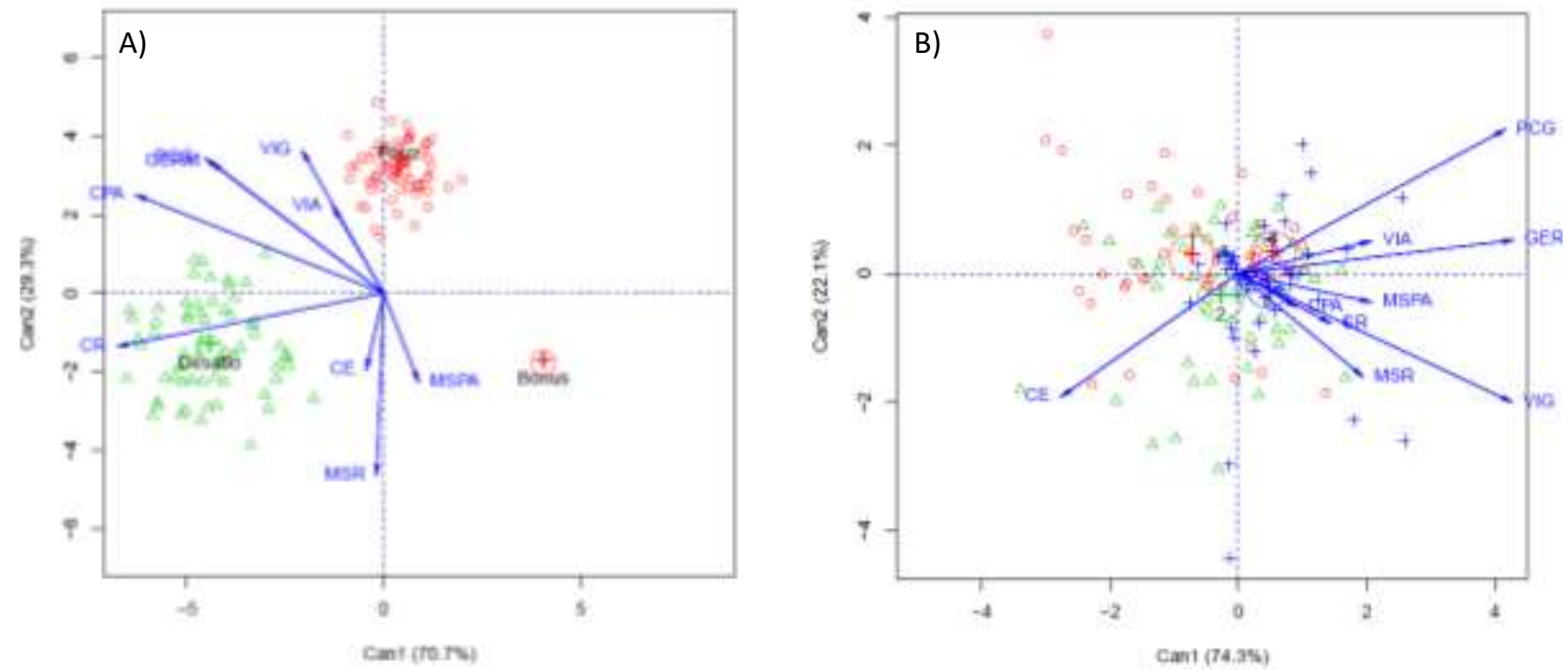

Fonte: Dados da pesquisa.

Na Figura 2B observa-se o efeito dos biológicos empregados na qualidade de sementes de soja. A maior associação das variáveis ligadas a qualidade de sementes se manifestou ligado aos tratamentos T4 e T3. O T4 (combinação de todos os biológicos empregados) promoveu a maior magnitude dos escores das variáveis PCG, GERM e VIA (em menor magnitude). O T3 (T. asperrellum) esteve associado aos maiores escores da variável VIG e em menor magnitude com MSPA, MSR, CPA e $\mathrm{CR}$, confirmando assim que o T. asperrellum promoveu um melhor desenvolvimento das plantas associada a um melhor vigor delas. Carvalho Filho et al. (2018) e Marra et al. (2019) demostraram que espécies de Trichoderma além de atuarem como promotores de crescimento de plantas favorecem o desenvolvimento das plantas aumentando a produtividade de sementes, corroborando o resultado obtido na presente pesquisa. Um resultado a destacar (não esperado) é a ausência de associação do T1 (controle) em relação a maior associação obtida para T4. Este resultado manifesta que a combinação dos biológicos foi a melhor opção para se obter a melhor qualidade de sementes nas condições avaliadas.

Analisando de uma forma conjunta todas as respostas obtidas, podemos afirmar que o comportamento das três cultivares de soja empregadas foi variável e determinou a resposta obtida, sendo a cultivar Brasmax Bônus IPRO quem manifestou os menores padrões de qualidades de sementes. Esta cultivar tem maior ciclo se comparada com as outras duas, possivelmente, a perda de qualidade pode ser atribuída ao maior tempo da cultura no campo. Mas tempo no campo representa maior exposição a condições adversas de clima e de pragas e doenças, redundando na perda de qualidade da cultivar Brasmax Bônus IPRO. Em relação aos biológicos empregados a inoculação de A. brasiliense e B. japonicum (T1) nas condições testadas 
não teve um lugar de destaque dentre as respostas obtidas, manifestando-se para algumas das variáveis inferior aos outro biológicos empregados (T2 e T3) ou a combinações de todos eles (T4).

Rego et al. (2018) verificou que a co-inoculação de B. japonicum e A. brasiliense melhorou o desenvolvimento e foi benéfico à qualidade fisiológica das sementes de soja produzidas, aumentando o percentual da primeira contagem de germinação, germinação e no teste de tetrazólio melhorou o vigor e a viabilidade das sementes. A co-inoculação de soja com Bradyrhizobium spp. e Azospirillum brasiliense favorece o desenvolvimento da nodulação precoce de plantas de soja (Chibeba et al., 2015), permitindo melhor crescimento e desenvolvimento devido ao aumento da disponibilidade de $\mathrm{N}$ pela fixação biológica do nitrogênio (Ferri et al., 2017). Estes resultados não coincidem com os obtidos no presente trabalho, e mostram em alguns resultados os outros tratamentos foram superiores a co-inoculação de A. brasiliense e B. japonicum (T1) (Tabela 4, Figura 2B).

Da Silva et al. (2020) mostram que o uso de Trichoderma sp. e B. japonicum líquido em sua forma isolada, proporcionam melhor desenvolvimento inicial para a cultura da soja, o que se evidencia como a resposta obtida ao combinar e usar isoladamente estes agentes biológicos na soja podem trazer respostas diferenciadas e dependentes dos ambientes e das cultivares empregadas. Essa combinação também foi efetiva no controle de Phytophtora sojae e crescimento de soja em campo (Azarmi et al., 2012), o que mostra o potencial desses biológicos que além de exercerem controle de doenças, podem promover o desenvolvimento das plantas e a qualidade das sementes obtidas.

\section{Conclusões}

Dentre as cultivares avaliadas Brasmax Foco IPRO e Brasmax Desafio IPRO apresentaram maior qualidade fisiológica. A cultivar Brasmax Bônus IPRO teve uma menor germinação, e nesse caso, o mix dos biológicos promoveu aumento na germinação e na qualidade fisiológica da soja.

A combinação dos biológicos (Azospirillum brasilense + Bradyrhizobium japonicum + BioAx + Trichoderma asperrellum) promove aumento na germinação e na qualidade fisiológica das sementes de soja que apresentam menor qualidade fisiológica. Em sementes de soja com alta qualidade fisiológica o efeito dos biológicos (isolados e combinados) não foi evidente.

\section{Agradecimentos}

Agradecemos à UFMS - CPCS, por garantir o espaço físico para a realização deste trabalho. Ao produtor Evandro Loeff e sua equipe, pela transferência do material biológico usado no experimento. Agradecer o apoio financeiro do Coordenador de Aperfeiçoamento de Pessoal de Nível Superior - Brasil (CAPES) aos Professores Visitantes JGA e AMZ. À Fundação Chapadão, por permitir o uso de máquinas e sementes para a implementação do experimento de campo.

\section{Referências}

Aguilera, J. G., Zuffo, A. M., Ratke, R. F., Alves, C. Z., Lima, R. E., Nogueira, G. A., \& da Silva Cândido, A. C. (2020a). Physiological quality of soybean seeds in response to doses of basalt powder. Research, Society and Development, 9(8), e840986314-e840986314. http://doi.org/10.33448/rsd-v9i8.6314

Aguilera, J. G., Zuffo, A. M., Ratke, R. F., Trento, A. C. S., Lima, R. E., Gris, G. A., \& Martins, W. C. (2020b). Influência de dosis de polvo de basalto sobre cultivares de soya. Research, Society and Development, 9(7), e51973974-e51973974. http://doi.org/10.33448/rsd-v9i7.3974

Aguilera, J. G., Zuffo, A. M., Ratke, R. F., da Silva, N. P., Marques, K. G. A., da Silva, L. N., Trento, A. C. S. \& Bernardo, J. T. (2020c). Respostas de componentes produtivos de soja a inoculação de biológicos em campo. Ensaios e Ciência, 24(4), 576-583. https://doi.org/10.17921/1415-6938.v24nespecial

AMK (2019) AMK orgânicos e biológicos: Benefícios do uso do Bio-Ax. Disponível em: http://amkbio.com.br. Acesso em: 13 out. 2019.

APPS (2013). Padrões para a produção e a comercialização de sementes de soja (Glycine max L.). Instrução Normativa n. 45 de 17 de Setembro de 2013 . In: Padrões de identidade e qualidade para produção e comercialização de sementes. Associação Paulista dos Produtores de Sementes e Mudas. 1-2. 
Azarmi, R., Hajieghrari, B. \& Giglou, A. (2011). Effect of Trichoderma isolates on tomato seedling growth response and nutrient uptake. African Journal of Biotechnology, 10(31), 5850-5855. https://doi.org/10.5897/AJB10.1600

Basal, O. \& Szabó, A. (2019). Inoculation enhances soybean physiology and yield under moderate drought. LIFE: International Journal of Health and LifeSciences, 5(2), 1-13. https://doi.org/10.20319/lijhls.2019.52.0113

Bhering, L. L. (2017). Rbio: A Tool For Biometric And Statistical Analysis Using The R Platform. Crop Breeding and Applied Biotechnology, 17, 187-90. https://doi.org/10.1590/1984-70332017v17n2s29

Brum, M. D. S., Martin, T. N., Cunha, V. S. D., Grando, L. F. T. \& Schonell, A. T. (2018). Soybean cultivation in a crop-livestock system with Azospirillum brasilense inoculation. Revista Caatinga, 31(1), 1-8. http://doi.org/10.1590/1983-21252018v31n101rc

Cadore, L. S., Vey, R. T., de Fresinghelli, J. C. F., Dotto, L., Mendes, F. B. \& da Silva, A. C. F. (2020). Trichoderma and Bradyrhizobium japonicum bioformulates on soy initial growth. Ciência e Natura, 42, 22. http://doi.org/10.5902/2179460X40873

Carvalho Filho, M. R., Martins, I., Peixoto, G. H. S., Muniz, P. H. P. C., Carvalho, D. D. C. \& de Mello, S. C. M. (2018). Biological control of leaf spot and growth promotion of Eucalyptus plants by Trichoderma spp. Journal of Agricultural Science, 10(9), 459-467. https://doi.org/10.5539/jas.v10n9p459

Cassán, F. \& Diaz-Zorita, M. (2016). Azospirillum sp. in current agriculture: From the laboratory to the field. Soil Biology and Biochemistry, 103, 117-130. https://doi.org/10.1016/j.soilbio.2016.08.020

Chibeba, A. M., Guimarães, M. D. F., Brito, O. R., Nogueira, M. A., Araujo, R. S. \& Hungria, M. (2015). Co-inoculation of soybean with Bradyrhizobium and Azospirillum promotes early nodulation. American Journal of Plant Sciences, 6, 1641-1649. http://doi.org/10.4236/ajps.2015.610164

CONAB (2020). Acompanhamento da safra brasileira de grão V. 7 - Safra 2019/20- N. 12 - Décimo segundo levantamento. 1-68.

Coradi, P. C., Milane, L. V., Camilo, L. J., Prado, R. L. F. \& Fernandes, T. C. (2015). Qualidade de grãos de soja armazenados em baixas temperaturas/quality of soybean grains stored in low temperatures. Revista Brasileira de Engenharia de Biossistemas, 9(3), 197-208. http://doi.org/10.18011/bioeng2015v9n3p197208

Cruz, C. D. (2013). Genes: a software package for analysis in experimental statistics and quantitative genetics. Acta Scientiarum. Agronomy, 35(3), 271-276. http://doi.org/10.4025/actasciagron.v35i3.21251

Cruz, C. D., Regazzi, A. J. \& Carneiro, P. C. S. (2004). Modelos biométricos aplicados ao melhoramento genético. UFV. 480p.

da Silva, V. T., Gava, R., Cotrim, M. F., Wassolowski, C. R., Teodoro, P. E. \& Snyder, R. L. (2020). Irrigation management in soybean culture under notillage system, on Brachiaria ruziziensis cultural remains. Research, Society and Development, 9(6), 64963430. http://doi.org/10.33448/rsd-v9i6.3430

Dias, M. A. N., Pinto, T. L. F., Mondo, V. H. V., Cicero, S. M. \& Pedrini, L. G. (2011). Direct effects of soybean seed vigor on weed competition. Revista Brasileira de Sementes, 33(2), 346-351. http://doi.org/10.1590/S0101-31222011000200017

dos Santos, C. E., Dias Neto, J. J., Neves, P. R., Fonseca, R. S. A., Kraemer, A. P. N. \& Paz-Lima, M. L. (2019). Tratamento químico de sementes e impacto sobre complexos de fitossanitários e elementos morfoagronômicos. Brazilian Applied Science Review, 3, 1445-1454. https://doi.org/10.34115/basrv3n2-049

dos Santos, E. R., Barros, H. B., Ferraz, E. C., Cella, A. J. S., Capone, A., Santos, A. F. \& Fidelis, R. R. (2011). Divergência entre genótipos de soja, cultivados em várzea irrigada. Revista Ceres, 58(6), 755-764. https://dx.doi.org/10.1590/S0034-737X2011000600012

Ferreira, L. L., Moreira, N. V., Carvalho, I. R., Santos, N. S. C. dos., Fernandes, M. de S., Pereira, A. I. de A., Curvêlo, C. R. da S., Silva, R. V. da., Amaral, U. do. \& Silva, R. de A. (2020). Spacing between rows of soybean genotypes: averages, regressions and correlations in yield levels. Research, Society and Development, 9(9), e952997793. https://doi.org/10.33448/rsd-v9i9.7793

Ferri, G. C., Braccini, A. L., Anghinoni, F. B. G. \& Pereira, L. C. (2017). Effects of associated co-inoculation of Bradyrhizobium japonicum with Azospirillum brasilense on soybean yield and growth. African Journal of Agricultural Research, 12(1), 6-11. http://doi.org/10.5897/AJAR2016.11711

França Neto J. B., Carrão-Panizzi M. C., Mandarino J. M. G. \& Krzyzanowski F. C. et al. (2013). Isoflavone contents in soybean seed subjected to harvest delay. In: Ista Seed Symposium, 119-120.

França Neto J. B., Krzyzanowski F. C. \& Henning A. A. (2010). A importância do uso de semente de soja de alta qualidade. Inf. Abrates 20: 37-38.

França Neto, J. B. \& Krzyzanowski, F. C. (2018). Documentos 406: Metodologia do teste de tetrazólio em sementes de soja. Londrina: Embrapa Soja, 94.

Galindo, F. S., Teixeira Filho, M. C. M., Buzetti, S., Ludkiewicz, M. G. Z., Rosa, P. A. L. \& Tritapepe, C. A. (2018). Technical and economic viability of coinoculation with Azospirillum brasilense in soybean cultivars in the Cerrado. Revista Brasileira de Engenharia Agrícola e Ambiental,22(1), 51-56. http://doi.org/10.1590/1807-1929/agriambi.v22n1p51-56

Haddad, P. E. (2014). Avaliação de isolados de Trichoderma spp. para o controle de Sclerotinia sclerotiorum e Meloidogyne incognita em soja e produção em meios líquidos.

Hungria, M., Campo, R. J. \& Mendes, I. C. (2007). A importância do processo de fixação biológica do nitrogênio para a cultura da soja: componente essencial para a competitividade do produto brasileiro. Londrina, PR: Embrapa Soja-Documentos (INFOTECA-E).

Jarecki, W. \& Bobrecka-Jamro, D. (2019). Influence of Seed Inoculation with Commercial Bacterial Inoculants (Bradyrhizobium Japonicum) on Growth and Yield of Soybean. Legume Research-An International Journal, 42(5), 688-693. http://doi.org/10.18805/LR-485 
Research, Society and Development, v. 10, n. 4, e52710414419, 2021

(CC BY 4.0) | ISSN 2525-3409 | DOI: http://dx.doi.org/10.33448/rsd-v10i4.14419

Khaledi, N. \& Taheri, P. (2016). Biocontrol mechanisms of Trichoderma harzianum against soybean charcoal rot caused by Macrophomina phaseolina. Journal of plant protection research, 56(1), 21-31. http://doi.org/10.1515/jppr-2016-0004

Krzyzanowski, F. C. (2004). Desafios tecnológicos para a produção de semente de soja na região tropical brasileira. In World Research Conference, 7, 13241335 .

Marcos Filho, J., CÍCERO, S. M. \& Silva, W. D. (1987). Avaliação da qualidade das sementes. Fealq.

Marra, R., Lombardi, N., d'Errico, G., Troisi, J., Scala, G., Vinale, F., Woo, S. L., Bonanomi, G. \& Lorito, M. (2019). Application of Trichoderma strains and metabolites enhances soybean productivity and nutrient content. Journal of agricultural and food chemistry, 67(7), 1814-1822. http://doi.org/10.1021/acs.jafc.8b06503

Mesquita, D. C. M., Ferreira, F. A., Martins, I., Mello, S. C. M. \& Carvalho, D. D. C. (2017). Antagonismo in vitro de Trichoderma spp. a Sclerotinia sclerotiorum do feijão comum. Agropecuária Científica no Semiárido, 13(1), 1-4, https://www.alice.cnptia.embrapa.br/bitstream/doc/10 74076/1/71033271PB.pdf

Pereira, A. S., Shitsuka, D. M., Parreira, F. J., \& Shitsuka, R. (2018). Metodologia da pesquisa científica. [e-book]. Santa Maria. Ed. UAB/NTE/UFSM. Recuperado de https://repositorio.ufsm.br/bitstream/handle/1/15824/Lic_Computacao_MetodologiaPesquisa-Cientifica.pdf?sequence=1 .

Rego, C. H. Q., Cardoso, F. B., da Silva Cândido, A. C., Teodoro, P. E. \& Alves, C. Z. (2018). Co-inoculation with Bradyrhizobium and Azospirillum Increases Yield and Quality of Soybean Seeds. Agronomy Journal, 110(6), 2302-2309. https://doi.org/10.2134/agronj2018.04.0278

Rondina, A. B. L., dos Santos Sanzovo, A. W., Guimarães, G. S., Wendling, J. R., Nogueira, M. A. \& Hungria, M. (2020). Changes in root morphological traits in soybean co-inoculated with Bradyrhizobium spp. and Azospirillum brasilense or treated with A. brasilense exudates. Biology and Fertility of Soils, 56, 537-549. https://doi.org/10.1007/s00374-020-01453-0

Saturno, D. F., Cerezini, P., da Silva, P. M., de Oliveira, A. B., de Oliveira, M. C. N., Hungria, M. \& Nogueira, M. A. (2017). Mineral nitrogen impairs the biological nitrogen fixation in soybean of determinate and indeterminate growth types. Journal of Plant Nutrition, 40(12), 1690-1701. https://doi.org/10.1080/01904167-2017-1310890

Soltani, N., Shropshire, C. \& Sikkema, P. H. (2006). Control of volunteer glyphosate-tolerant maize (Zea mays) in glyphosate-tolerant soybean (Glycine max). Crop Protection, 25(2), 178-181. https://doi.org/10.1016/j.cropro.2005.03.017

Tamagno, S., Sadras, V. O., Haegele, J. W., Armstrong, P. R. \& Ciampitti, I. A. (2018). Interplay between nitrogen fertilizer and biological nitrogen fixation in soybean: implications on seed yield and biomass allocation. Scientific reports, 8(1), 1-11. https://doi.org/10.1038/s41598-018-35672-1

Zambiazzi, E. V., Godinho, S. H. M., Mendes A. E. S., Ribeiro., F. O., Zuffo, A. M., Lima, J. G., \& Carvalho, M. L. M. (2017). Estimates of genetics and phenotypics parameters for the yield and quality of soybean seeds. Genetics and Molecular Research, 16(3), gmr16039801. https://doi.org/10.4238/gmr16039801

Zuffo, A. M., Bruzi, A. T., de Rezende, P. M., de Carvalho, M. L. M., Zambiazzi, E. V., Soares, I. O. \& Silva, K. B. (2016). Foliar application of Azospirillum brasilense in soybean and seed physiological quality. African Journal of Microbiology Research, 10(20), 675-680. https://doi.org/10.5897/AJMR2016.7911 